\title{
Optimization and analysis for palm oil mill operations via input-output optimization model
}

\author{
Steve Z. Y. Foong ${ }^{1}$, Viknesh Andiappan ${ }^{2}$, Raymond Tan ${ }^{3}$, and Denny K. S. $\mathrm{Ng}^{1, *}$
}

\author{
${ }^{1}$ Department of Chemical and Environmental Engineering/Centre of Sustainable Palm Oil Research (CESPOR), The \\ University of Nottingham Malaysia Campus, Broga Road, Semenyih 43500, Malaysia \\ ${ }^{2}$ School of Engineering and Physical Sciences, Heriot-Watt University Malaysia, 62200, Putrajaya, Wilayah Persekutuan \\ Putrajaya, Malaysia \\ ${ }^{3}$ Centre for Engineering and Sustainable Development Research, De La Salle University, 2401 Taft Avenue, 1004 \\ Manila, Philippines
}

\begin{abstract}
A typical palm oil mill produces crude palm oil, crude palm kernel oil and other biomass from fresh fruit bunches. While the milling process is well established in the industry, insufficient research and development has been done on analyzing the operational performance of a mill. Factors such as operating time and fruit availability affect the performance of a palm oil mill (e.g., capital, operating and labor costs). This paper presents an input-output model to optimize the operations of a palm oil mill based on maximum economic performance. Following this, feasible operating range analysis (FORA) is performed to study the utilization and flexibility of the process. A palm oil mill case study in Malaysia is used to illustrate the proposed approach. Based on the optimized results, it was found that $37 \%$ reduction in capital cost and $49 \%$ increase in economic performance is achieved. Meanwhile, the utilization index of the mill during peak season increases from 0.48 to 0.76 .
\end{abstract}

\section{Introduction}

Oil palm (Elaeis guineensis) is grown for production of palm oil products due to its stability, high yield and low cost $[1,2]$. It has been the most widely used vegetable oil to fulfil the global demand for oils and fats [3]. Crude palm oil (CPO) is extracted from fresh fruit bunches (FFBs) in a processing facility known as palm oil mill (POM).

A typical milling process consists of several operational units as shown in Fig. 1. FFBs undergo sterilization, threshing, digestion and pressing to produce pressed liquid and cake. The produced pressed liquid is clarified and purified to produce CPO, while pressed cake undergoes nut separation, nut cracking, kernel separation and drying to produce palm kernel (PK). Throughout the milling process, biomass such as palm kernel shell (PKS), empty fruit bunch (EFB) and palm pressed fiber (PPF) are generated as by-products. Meanwhile, strong wastewater known as palm oil mill effluent (POME) is also generated during sterilization and clarification operations.

In order to minimize logistics costs, POMs are located near plantations in remote areas. As a result, these POMs are typically positioned far away from the electrical grid [4]. This leaves POMs at a disadvantage, as they would require steam and electricity for $\mathrm{CPO}$ extraction. In current practice, the process steam and electricity demands are usually fulfilled by burning PPF and a portion of PKS generated from the milling process via co-generation system. Excess PKS is sold as alternative solid fuel, while EFB is pressed for oil recovery [5] before being returned to plantations as mulch [6] or composted to produce biofertilizer. Meanwhile, pond-based systems are used to treat POME prior to discharge.

The processing capacity of a plant or system depends on the space, labor, equipment, technology and materials available [7]. In this sense, POMs would have their own unique design features and the operations of each mill may differ between one another. Ripe FFBs collected from plantations must be transported and processed immediately in POMs to prevent degradation of $\mathrm{CPO}$ quality due to increased free fatty acid content [8]. The amount of FFBs supplied to a POM could vary depending on location and time, due to seasonal changes and/or possible unforeseen circumstances in the plantations $[9,10]$. To overcome this issue, palm oil millers tend to build POM with excess capacity to ensure higher flexibility [11] and lower processing cost [12]. This however, affects the utilization and economic performance of POM, especially during lean crop season.

In this work, an input-output optimization model (IOM) is utilized to optimize and analyze the interdependencies between operational units, material flows and costs in a palm oil milling process. Apart from Foong et. al [10], limited work has been done on the optimization of palm oil milling process. Furthermore, operational parameters and variables such as operating hours, labor costs, process utilization and flexibility were not considered previously.

*Corresponding author: Denny.Ng@nottingham.edu.my 
As such, this work considered the mentioned aspects. Alongside this, variation in FFB availability and its corresponding impact on operations, costs, utilization and flexibility indices are considered in optimizing the milling process.

In the following section, the problem statement for this work is presented, followed by a detailed formulation for IOM in Section 3. Next, an existing POM flowsheet is optimized using the input-output approach described in Section 4. Following this, the economic performance, utilization and flexibility of the POM is then compared to highlight the improvements achieved. Lastly, the conclusions and future works are described in the remaining sections.

\section{Problem statement}

The problem addressed by the proposed model is formally stated as follows. Palm oil milling process is expressed in an input and output model with $\mathrm{a}_{m t e}$ for material $m \in \mathrm{M}$ input and output, to and from technology te $\epsilon$ TE. For each crop season $s$, the number of units operated for technology, $U_{t e}$ is determined from the nominal capacity, $\mathrm{CAP}_{t e}$ available in the market. Each material $m \in \mathrm{M}$ and technology te $\epsilon$ TE are associated with a given material cost, $\mathrm{C}_{m}$, operating cost, $\mathrm{OC}_{t e}$ and capital costs, $\mathrm{CC}_{t e}$, respectively. The objective is to maximize the economic performance, $E P$ of the POM as shown in Eq. (1).

Maximize EP

\section{Input-output optimisation model (IOM) formulation}

In the model, the index $s$ represents the crop season in which a material flows would vary. It is assumed that the ratios of material flows remain constant and a linear correlation for material flows in the milling process is given in Eq. (2)

$$
{ }_{t e=1}^{\mathrm{TE}}\left(\mathrm{a}_{m t e} x_{t e}\right)_{s}=\left(y_{m}\right)_{s} \quad \forall \mathrm{m}, \forall \mathrm{s}
$$

where $\mathrm{a}_{m \text { te }}$ represents the input and output ratios of material $m$, to and from technology te. $\mathrm{a}_{m t e}$ is expressed in negative value for material inputs, positive value for material outputs or zero if there are no interactions between material $m$ and technology te. Meanwhile, $x_{t e}$ is the processing capacity of technology te, which is expressed in positive values when technologies are operated or zero when it is not. $y_{m}$ is the net flowrate of a given material $m$ (i.e., net input or output) where final and by-products are indicated with positive values. Process feedstocks are indicated with negative values while intermediates are denoted with zeros.

Electricity $e$ is considered as one of the materials $m$ produced in the process. It is also being consumed to operate technology te for material conversions. However, electricity demand, $E^{\text {Demand }}$ of a POM relies on the number of units operated for technology, $U_{\text {te }}$ as shown in Eq. 3:

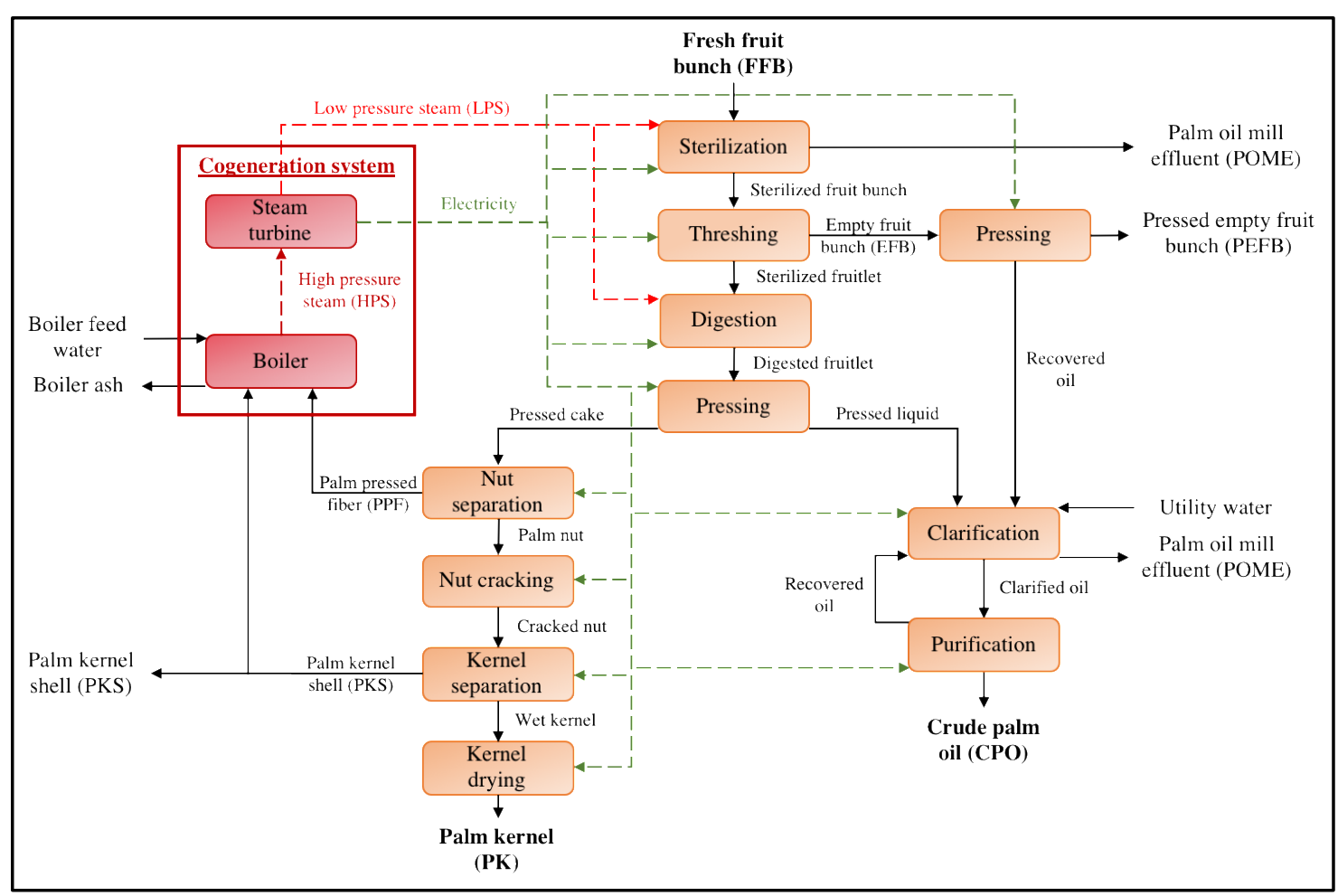

Figure 1. Unit operations in typical palm oil mill. 


$$
\left(E^{\text {Demand }}\right)_{s}=\left(\sum_{t e=1}^{\mathrm{TE}} U_{t e} \mathrm{E}_{t e}\right)_{s} \quad \forall s
$$

where $\mathrm{E}_{t e}$ is the electricity consumption specified per unit technology te operated. As shown in Eq. (4), number of units of technology operated, $U_{t e}$ is determined based on the nominal capacity, $\mathrm{CAP}_{t e}$ available in the market

$$
\left(x_{t e}\right)_{s} \mathrm{CAP}_{t e}^{-1}\left(U_{t e}\right)_{s} \quad \forall \mathrm{s}, \forall \mathrm{e}
$$

and $\mathrm{CAP}_{t e}^{-1}$ is the inverse matrix of nominal capacity, $\mathrm{CAP}_{t e}$. Note that the units of technology operated, $U_{t e}$ are positive integers, subject to Eq. (5)

$$
\left(U_{t e}\right)_{s}\left(\mathrm{U}_{t e}^{\max }\right)_{s} \quad \forall s
$$

where $\mathrm{U}_{\text {te }}^{\mathrm{max}}$ is the maximum units of technology installed. To ensure that the process is self-sufficient without interruption, an additional constraint, Eq. (6) is included whereby the output of electricity produced, $y_{\mathrm{e}}$ in the process is greater or equal to the electricity demand, $E^{\text {Demand }}$.

$$
\left(y_{e}\right)_{s}\left(E^{\text {Demand }}\right)_{s} \quad \forall s
$$

Meanwhile, Eq. (7) evaluates the economic performance $E P$ of the process

$$
E P=G P \quad C R F \quad C A P E X
$$

where GP, CRF and CAPEX represents the gross profit, capital recovery factor and capital costs required, respectively. GP can be calculated using Eq. (8)

$$
G P=A O T \sum_{s} s\left[\left(\sum_{m=1}^{\mathrm{M}} y_{m} \mathrm{C}_{m}+O P E X+O T C\right)_{s}+L C\right]
$$

whereby $A O T, \alpha_{s}, \mathrm{C}_{m}, O P E X, O T C$ and $L C$ are the annual operational time, fraction of occurrence, material, total operating, over time and labor costs, respectively.

$C R F$ is used to annualize CAPEX over a specified operation lifespan $\mathrm{t}_{t e}^{\max }$ and discount rate, r. $C R F$ can be determined via Eq. (9).

$$
C R F=\frac{\mathrm{r}(1+\mathrm{r})^{\mathrm{t}_{\text {max }}}}{(1+\mathrm{r})^{\mathrm{t}_{\text {max }}^{\text {max }}}} 1
$$

CAPEX and OPEX are calculated based on the maximum units of technology installed, $\mathrm{U}_{t e}^{\max }$ and units of technology operated, $U_{t e}$ in the process as shown in Eqs. (10-11).

$$
\begin{gathered}
C A P E X={ }_{t e=1}^{\mathrm{TE}} \mathrm{U}_{t e}^{\mathrm{max}} \mathrm{CC}_{t e} \\
(O P E X)_{s}={ }_{t e=1}^{\mathrm{TE}} U_{t e} \mathrm{OC}_{t e}
\end{gathered}
$$

$\mathrm{CC}_{t e}$ and $\mathrm{OC}_{t e}$ represent the capital and operating costs per unit of technology te. Meanwhile, OTC and $L C$ are calculated via Eqs. (12-13)

$$
\begin{aligned}
& (O T C)_{s}=\mathrm{C}_{\mathrm{OT}} n_{\mathrm{wk}}\left[(A O T)_{s} \mathrm{AST}\right] \quad \forall s \\
& L C=\mathrm{C}_{\mathrm{lab}} \mathrm{n}_{\mathrm{wk}} \mathrm{n}_{\mathrm{ws}}
\end{aligned}
$$

whereby $\mathrm{C}_{\mathrm{OT}}$ and $\mathrm{C}_{\mathrm{lab}}$ are the overtime cost and labor cost. $\mathrm{n}_{\mathrm{wk}}$ and $\mathrm{n}_{\mathrm{ws}}$ represents the number of workers and working shifts per day while AST is the annual shift time of the process.

To illustrate the proposed approach, a typical POM case study adopted from the previous study [10] is presented in the next section.

\section{Case study}

The palm oil milling process developed by Foong et. al [10] is adopted as the baseline POM design in this case study. It is assumed that the mill operator is interested to optimize the milling process to achieve higher economic performance, $E P$ by taking operational factors such as operating hours, labor costs, and FFB availability into account. Three crop seasons, i.e. low, medium and high were considered with the given fractions of occurrence, $\alpha_{s}$ and FFB availability shown in Table 1.

Table 1. Fraction of occurrence and FFB available with respect to crop seasons [10].

\begin{tabular}{lcc}
\hline Crop season & $\begin{array}{c}\text { Fraction of occurrence, } \\
\boldsymbol{\alpha}_{\boldsymbol{s}}\end{array}$ & $\begin{array}{c}\text { FFB availability } \\
(\mathbf{t} / \mathbf{y})\end{array}$ \\
\hline Low & $\alpha_{\mathrm{L}}=0.417$ & 195,800 \\
Medium & $\alpha_{\mathrm{M}}=0.333$ & 261,000 \\
High & $\alpha_{\mathrm{H}}=0.250$ & 369,800 \\
\hline Average & & 236,000 \\
\hline
\end{tabular}

A typical POM operates in batches for 12 hours daily, usually divided into 2 workings shifts (i.e. annual shift time, AST $=4,350 \mathrm{~h} / \mathrm{y}$ ). 15 operators with a labor cost, $\mathrm{C}_{\mathrm{lab}}$ of 4,500 USD/y are required for each shift to operate the milling process. It is also assumed that the POM will have an operation lifespan, $\mathrm{t}_{t e}^{\max }$ of 15 years with a discount rate, $\mathrm{r}$ of $5 \%$ per annum. Detailed information on economic parameters, material flows, and technology units operated for the baseline POM design were summarized in Tables S1 - S3. Note that the technology units operated during high crop season, $U_{\mathrm{H}}$ (Table $\mathbf{S 3}$ ) is equal to the maximum units of technology installed, $\mathrm{U}_{t e}^{\max }$. The process matrix table and other specifications such as $\mathrm{CAP}_{t e}, \mathrm{E}_{t e}, \mathrm{CC}_{t e}, \mathrm{OC}_{t e}$ and $\mathrm{C}_{m}$ of the POM are presented in Tables $\mathbf{S 4}-\mathbf{S 6}$ under the Supporting Information section.

It was found that the baseline POM design did not efficiently utilize its capacity. The assumption that milling process can only be operated for 4,350 hours a year due to the working shifts of operators causes its capacity to be underutilized. As a result, more technology units must be installed to process all the FFBs supplied to prevent degradation in CPO quality, especially during high crop season. This shows a limitation in the previous study which should be considered in optimizing a palm oil 
milling process. The common practice in the industry is to increase the annual operating time, $A O T$ of the process. In the industry, POM may operate up to 19 hours per day or 7,000 hours yearly $(A O T \leq 7,000)$. In that sense, the total capital costs, CAPEX needed can be reduced as lesser technology units are required. However, the increment in $A O T$ on top of $4,350 \mathrm{~h} / \mathrm{y}$ AST requires overtime cost, $O T C$ to be paid for operators working extra time and operating costs, $\mathrm{OC}_{t e}$ for service and maintenance of technology units. An additional of $5 \mathrm{USD} / \mathrm{h}$ overtime costs, $\mathrm{C}_{\mathrm{OT}}$ and $20 \%$ for $\mathrm{OC}_{t e}$ are considered for operations more than 4,350 hour annually.

In order to achieve higher economic performance, $E P$ in the POM, an IOM (shown in Tables S4 - S6) was developed based on Eqs. $(1$ - 13). The developed mixed integer linear programming (MILP) model was solved via LINGO v14, with Global [13], with an Intel ${ }^{\circledR}$ Core $^{\mathrm{TM}}$ i5 (2 x $3.20 \mathrm{GHz}$ ), 8 GB DDR3 RAM desktop unit. Following that, feasible operating range analysis (FORA) [14] is performed on the POM to determine the operational range (i.e., the minimum, $y_{m}^{\min }$ and maximum, $y_{m}^{\max }$ net output flowrates) of CPO product. Subsequently, utilization index, $U I$ and flexibility index, FI were quantified via Eqs. $(14$ - 15) to analyze the operational performance of the POM.

$$
\begin{gathered}
U I=\frac{y_{m}}{y_{m}^{\max }} \\
F I=\frac{y_{m}^{\max } y_{m}}{y_{m}^{\max }}
\end{gathered}
$$

$U I$ and $F I$ ranges between zero to one. In the event where $U I$ equals to zero, the process is not being utilized while $U I$ equals to one indicates that the process is operating at $100 \%$ of the processing capacity installed. Meanwhile, zero in FI represents that the process has no flexibility in its operation and vice versa.

\section{Results and discussions}

The baseline POM presented in Tables S1 - S3 is optimized using the objective in Eq. (1), subject to Eqs. (2 -13). The IOM consists of 139 continuous variables with 18 integer variables and 219 constraints, solved in 1.3 seconds to achieve a global solution. The results showed that optimized POM was able to achieve an EP of 5.01 million USD/y (32.5\% increment) as compared to 3.78 million USD/y obtained previously. The remaining economic parameters for the optimized POM design are summarized in Table 5. Detailed information on material flows and technology units operated are delivered under the Supporting Information section (Table S7 - S8).
Table 2. Economic parameters for optimized POM design.

\begin{tabular}{lcccc}
\hline & \multicolumn{4}{c}{ Crop season } \\
\cline { 2 - 5 } Economic parameters & Low & Medium & High & $\begin{array}{c}\text { Averag } \\
\mathrm{e}\end{array}$ \\
\hline Annual operational time, AOT (h/y) & 5,640 & 7,519 & 6,656 & 6,519 \\
Total capital costs, CAPEX (million & & 11.59 & & 11.59 \\
USD) & & -0.14 & & -0.14 \\
Labor costs, LC (million USD/y) & -0.10 & -0.24 & -0.17 & -0.16 \\
Overtime costs, OTC (million USD/y) & -0.79 & -0.92 & -1.17 & -0.92 \\
Total operating costs, OPEX (million & 4.54 & 6.01 & 8.92 & 6.12 \\
USD/y) & 3.42 & 4.18 & 7.80 & 5.01 \\
Gross Profit, GP (million USD/y) & & & & \\
Economic Performance, EP (million & USD/y) & &
\end{tabular}

CAPEX decreased from 18.42 to 11.59 million USD as the units of technology required, $\mathrm{U}_{t e}^{\max }$ was reduced from 39 (Table S3) to 27 units (Table S8). Note that the material flows do not vary much for both POM designs (Table S2 \& S7) due to the linear correlation in material flows given in Eq. (2). However, the average $E^{\text {Demand }}$ was reduced by $100 \mathrm{MWh} / \mathrm{y}$, resulting in an increment of 1,000 t PKS produced per year as lesser PKS was combusted for electricity generation. On the other hand, a longer AOT was required for the optimized POM design from 4,350 to $6,519 \mathrm{~h} / \mathrm{y}$ on average $(5,640,7,519$ and $6,656 \mathrm{~h} / \mathrm{y}$ for low, medium and high crop seasons, respectively). In this respect, additional $O T C$ by $0.10,0.24$ and 0.17 million USD/y was required for different crop seasons (an average of 0.16 million USD/y). Despite an increment in $\mathrm{OC}_{t e}$ by $20 \%$ due to longer $A O T, O P E X$ was reduced from 1.34 to 0.92 million USD/y on average, since the units of technology operated for all season decreases. The costs allocation for the baseline and optimized design are presented in Fig. 2 as solid and stripes bar, respectively (CAPEX is annualized to yearly basis). On average, $23 \%$ of the costs required by the baseline design was reduced while still achieving the same $\mathrm{CPO}$ output with a difference of 16,11 and $41 \%$ in total costs for low, medium and high crop seasons, respectively.

The operational performance of POM was quantified and analyzed using FORA framework to show the increment in CPO production capacity with respect to CAPEX invested. Fig. 3 presents the operational performance of a POM in terms of $U I$ (Eqs. (14)) and FI (Eqs. (15)) during high crop season. It was shown that the CPO production capacity increases in steps with CAPEX. Each step in Fig. 3 represents an increment in units of technology invested, rising the CPO production capacity in return. The baseline $\mathrm{POM}$ design has a $\mathrm{CPO}$ production capacity of $159,000 \mathrm{t} \mathrm{CPO/y,} \mathrm{which} \mathrm{is} \mathrm{more} \mathrm{flexible} \mathrm{(} F I=$ $0.52)$ than the optimized design $(F I=0.24)$ with a production capacity of $101,000 \mathrm{t} \mathrm{CPO} / \mathrm{y}$. However, this reduces the utilization of $\mathrm{POM}$ (i.e. $U I=0.76$ in optimized design to 0.48 in baseline design), showing that the equipment invested in the baseline design are not fully utilized to its maximum potential due to limited FFB availability. 


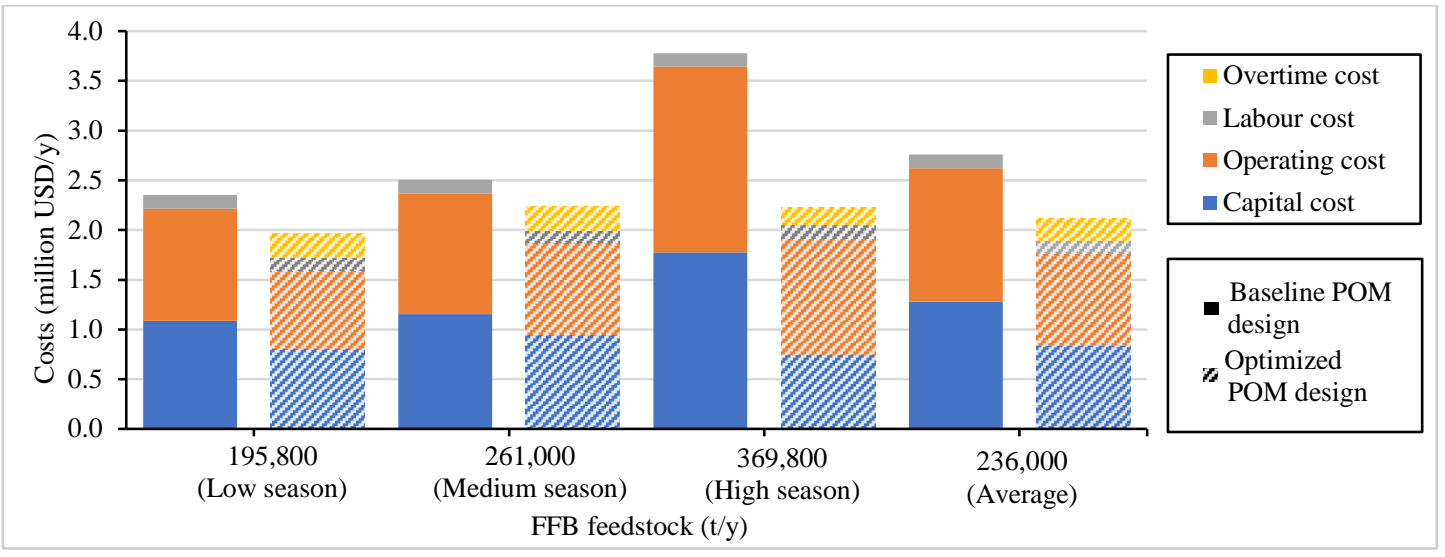

Figure 2. Costs allocation for baseline against optimized palm oil mill design.

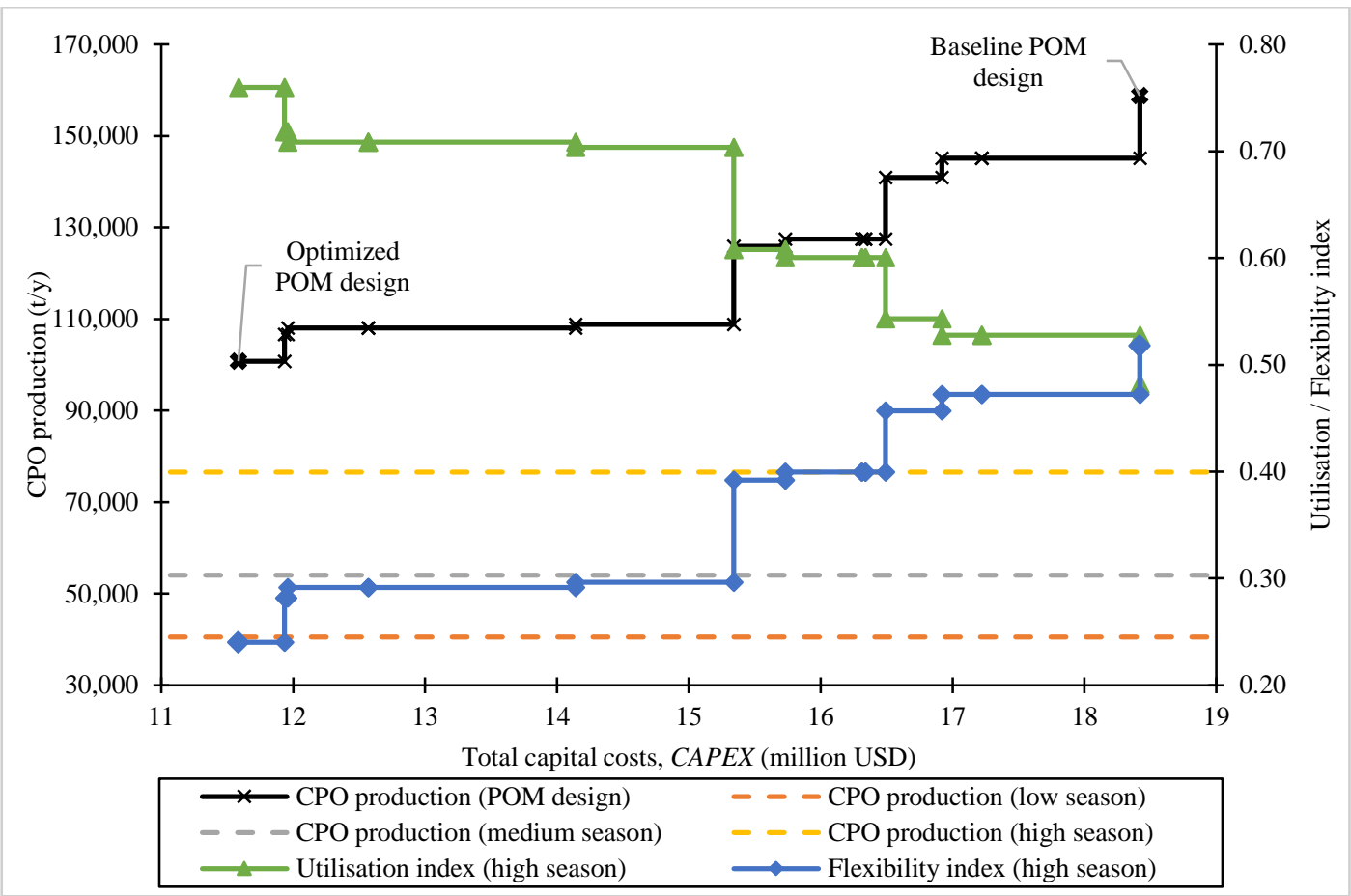

Figure 2. Feasible operating range analysis for palm oil mill.

\section{Conclusion}

In this work, an IOM for optimization of POM operations is presented. It is shown that a higher $E P$ is achieved from the optimized POM design generates as compared to the baseline design synthesized previously. Integration of downstream processes such as biorefinery by considering POM operations can be reflected as prospects for future works.

This research was financially supported by The Ministry of Higher Education, Malaysia (LRGS UPM Vot 5526100 and LRGS/2013/UKM-UNMC/PT/05).

\section{References}

[1] Petrenko C, Paltseva J, Searle S. Ecological Impacts of Palm Oil Expansion in Indonesia. Washington D.C.: 2016.
[2] Reeves JB, Weihrauch JL. Composition of Foods: Fats and Oils: Raw, Processed, Prepared. Agricultur. Washington D.C.: United States Department of Agriculture Science and Education Administration; 1979.

[3] European Palm Oil Alliance. What is Palm Oil? 2016. https://www.palmoilandfood.eu/en/what-ispalm-oil (accessed August 3, 2018).

[4] Aziz M, Oda T, Kashiwagi T. Design and Analysis of Energy-Efficient Integrated Crude Palm Oil and Palm Kernel Oil Processes. J Japan Inst Energy 2015;94:43-150.

[5] Gomez JC, Mokhtar MN, Sulaiman A, Baharuddin AS, Busu Z. Recovery of Residual Crude Palm Oil from the Empty Fruit Bunch Spikelets Using Environmentally Friendly Processes. Sep Sci Technol 2015;50:1677-83. doi:10.1080/01496395.2014.994781. 
[6] Elbersen J van D en W. Palm Oil Production for Oil and Biomass: The Solution for Sustainable Oil Production and Certifiably Sustainable Biomass Production? 2004.

[7] Yu-Lee RT. Essentials of Capacity Management. Wiley; 2002.

[8] Department of Environment. Industrial Processes \& The Environment (Handbook No. 3): The Crude Palm Oil Industry. 1999.

[9] Malaysian Palm Oil Board (MPOB). Yield 20172018. http://bepi.mpob.gov.my/index.php/en/statistic s/yield/181-yield-2017/817-yield-2017.html (accessed February 21, 2018).

[10] Foong SZY, Lam YL, Andiappan V, Foo DCY, $\mathrm{Ng}$ DKS. A Systematic Approach for the Synthesis and Optimization of Palm Oil Milling Processes 2018;57:2945-2955. doi:10.1021/acs.iecr.7b04788.

[11] Sharifzadeh M. Integration of Process Design and Control: A review. Chem Eng Res Des 2013;91:2515-49. doi:10.1016/J.CHERD.2013.05.007.

[12] Azman I. The Impact of Palm Oil Mills' Capacity on Technical Efficiency of Palm Oil Millers in Malaysia. Oil Palm Ind Econ J 2014;14:35-41.

[13] LINDO Systems Inc. LINGO The Modeling Language and Optimizer 2016.

[14] Andiappan V, Ng DKS, Tan RR. Design Operability and Retrofit Analysis (DORA) framework for energy systems. Energy 2017;134:1038-52.

doi:10.1016/j.energy.2017.06.054. 